\title{
Rapid effectiveness of certolizumab pegol in non-radiographic axial spondyloarthritis
}

\author{
A. Spadaro', A. Scarno ${ }^{1}$, A. Carboni' ${ }^{1}$, F.M. Perrotta ${ }^{1}$, \\ C. Catalano ${ }^{2}$, E. Lubrano ${ }^{3}$, G. Valesini ${ }^{1}$ \\ ${ }^{1}$ Dipartimento di Medicina Interna e Specialità Mediche, U.O.C. di Reumatologia, \\ Università "La Sapienza", Roma; \\ 2Dipartimento di Medicina Molecolare, Radiologia Centrale U.O.C. A, Università "La Sapienza", Roma; \\ ${ }^{3}$ Dipartimento di Medicina e di Scienze per la Salute, Università del Molise, Campobasso, Italy
}

\begin{abstract}
SUMMARY
In axial spondyloarthritis (SpA), the efficacy of certolizumab pegol (CZP), a novel pegylated anti-tumor necrosis factor alpha drug has not been investigated. We report that CZP showed a rapid effectiveness, assessed clinically and by magnetic resonance imaging, in a patient with a non-radiographic axial $\mathrm{SpA}$, classified according to Assessment in SpondyloArthritis International Society (ASAS) criteria. This case suggests that CZP could be considered an useful treatment in non-radiographic axial SpA, supporting that an earlier therapeutic approach could play a relevant role in the management of the disease.
\end{abstract}

Key words: Certolizumab pegol, Axial spondyloarthritis, Magnetic resonance.

Reumatismo, 2013; 65 (3): 134-137

\section{INTRODUCTION}

pondyloarthritis $(\mathrm{SpA})$ are a group of diseases that comprise ankylosing spondylitis, psoriatic arthritis, arthritis/ spondylitis with inflammatory bowel disease, reactive arthritis and undifferentiated $\mathrm{SpA}$, whereas patients with typical features of SpA do not fulfill the criteria for one of these subtypes. SpA patients can also be distinguished according to their clinical presentation as patients with predominantly peripheral $\mathrm{SpA}$ or with predominantly axial SpA. In axial SpA, radiographic changes of sacroiliac joints reflects structural damage, as a consequences of inflammation, while magnetic resonance imaging (MRI) can detect inflammatory lesions (i.e. bone marrow edema/osteitis) some years before the appearance of radiographic sacroiliitis (1). In this context, Assessment in SpondyloArthritis international Society (ASAS)

Corresponding author: Prof. Antonio Spadaro Dipartimento di Medicina Interna e Specialità Mediche U.O.C. di Reumatologia Università "La Sapienza", Roma Azienda Policlinico Umberto Viale del Policlinico, 155 - 00161 Roma, Italy E-mail: a.spadaro.reuma@virgilio.it criteria for axial $\mathrm{SpA}$ that include patients with and without radiographic sacroiliitis were developed (2).

Tumor necrosis factor (TNF) alpha antagonists, such as adalimumab, etanercept and infliximab have been used in the treatment of patients with SpA without radiographically defined sacroiliitis in open-label studies (3-5) and in randomized controlled trials (6-8) in order to ascertain the efficacy of these drugs.

Early SpA controlled trials, showed that infliximab (7) etanercept (8) and adalimumab (9) reduced active inflammation of sacroiliac joints detected by MRI, the best imaging method for the detection of active sacroilitis in patients with $\operatorname{SpA}(2)$. In axial $\mathrm{SpA}$, the efficacy of certolizumab pegol (CZP), a novel pegylated anti-TNF alpha drug approved for the treatment of adult patients with moderately to severely active rheumatoid arthritis, has not been investigated.

In this paper, we reported a case of a patient with non-radiographic axial SpA, classified according to ASAS criteria (2), treated with CZP that showed both a good clinical and MRI imaging response.

\section{CASE REPORT}

A 24-year old woman with SpA (disease duration $=22$ months), HLA-B27 posi- 
tive, was admitted to our rheumatologic unit for inflammatory lumbar and sacroiliac pain, swelling of right Achilles tendon, right knee arthritis associated with early morning stiffness lasting more than $60 \mathrm{~min}$. Her father had ankylosing spondylitis. The patient failed treatment with salazopyrine ( $2 \mathrm{~g} /$ day), non-steroidal antiinflammatory drugs and local injections of corticosteroids. For 13 months she received methotrexate $(15 \mathrm{mg} / \mathrm{i} . \mathrm{m} . /$ weekly $)$, 6-methylprednisolone ( $8 \mathrm{mg} /$ daily) and indomethacin $(150 \mathrm{mg} /$ daily). Clinical evaluation showed bath ankylosing spondylitis disease activity index $($ BASDAI $)=8$; bath ankylosing spondylitis functional index $(\mathrm{BASFI})=4.7$; bath ankylosing spondylitis metrology index $($ BASMI $)=0$, with modified Schöber test $=4.1 \mathrm{~cm}$ and lumbar side flexion $=10.5 \mathrm{~cm}$.

Laboratory exams showed: erythrocyte sedimentation rate $=8 \mathrm{~mm} / 1^{\text {st }} \mathrm{h}, \mathrm{C}$-reactive-protein $=3 \mathrm{mg} / \mathrm{L}$ and mild normocytic normochromic anaemia. Antinuclear antibodies, rheumatoid factor, anti citrullinated peptide antibodies and tuberculin skin test were negative. Pelvis $\mathrm{x}$-ray did not show signs of sacroiliitis. Pelvis MRI showed bone marrow edema of right sacroiliac joint on short tau inversion recovery sequences (STIR) (Fig. 1A), indicative of active sacroiliitis. Right ankle MRI showed thickening of Achilles tendon associated with hyperintense signal at the calcaneus insertion, due to bone marrow edema, and thickening of tibialis posterior and flexor digitorum longus tendons. After clinical, laboratory and imaging investigation, CZP
(400 mg subcutaneously at weeks 0,2 and 4 , followed by $200 \mathrm{mg}$ every 2 weeks) was added to methotrexate $(15 \mathrm{mg} / \mathrm{i} . \mathrm{m} . /$ weekly), 6-methylprednisolone $(8 \mathrm{mg} /$ daily $)$ and indomethacin $(150 \mathrm{mg} /$ daily $)$ treatment. One day after the first administration of CZP the patient reported a dramatic improvement of inflammatory back pain, knee arthritis and heel enthesitis. Her clinical improvement continued during the following weeks. After 4 weeks she withdrew methotrexate and indomethacin because of persistent nausea. The good response $(\mathrm{BASDAI}=0.6 ; \mathrm{BASFI}=1.9 ; \mathrm{BASMI}=$ 0 with modified Schöber test $=7 \mathrm{~cm}$ and lumbar side flexion $=18.5 \mathrm{~cm}$ ) permitted to taper corticosteroid treatment $(4 \mathrm{mg} /$ daily). On January 2012, after 12 weeks of CZP treatment, clinical response was maintained and MRI showed an important improvement of active sacroiliitis (Fig. 1B) and right ankle enthesitis (thickening and bone marrow edema at calcaneus insertion of right Achilles tendon were largely reduced).

\section{DIsCussion}

In axial SpA radiographic changes of sacroiliac joints reflects structural damage, as a consequences of inflammation. In fact radiographic sacroiliitis, an essential part of the widely accepted modified New York criteria for ankylosing spondylitis (10) appeared years after inflammatory lesions on MRI and the onset of inflammatory back pain. ASAS developed criteria for axial

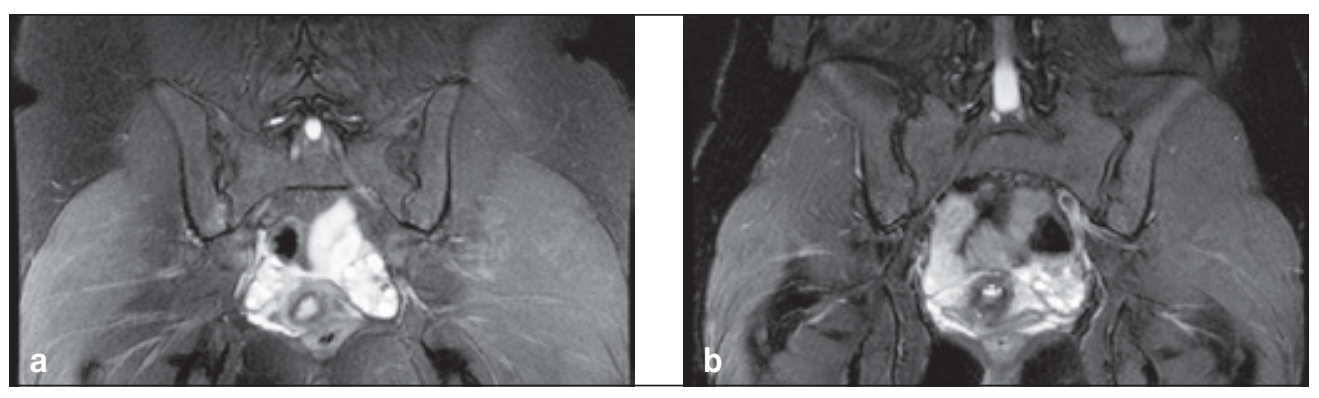

Figure 1 - Short tau inversion recovery sequences of sacroiliac joints. (A) At baseline, bone marrow edema of right sacroiliac joint appears as a focal hyperintensity on the iliac side. (B) After 12 weeks of certolizumab pegol treatment, the hyperintensity is largely reduced. 
SpA that include patients with established radiographic sacroiliitis and also patients in non-radiographic stage (2). In this context, active inflammation of the sacroiliac joints, as shown by MRI, is an important part of imaging arm of ASAS criteria for axial SpA. In fact, MRI can detect active inflammatory (bone marrow edema/osteitis, synovitis, enthesitis and capsulitis) and structural damage (sclerosis, erosions, fat deposition and ankylosis) lesions, but only the clear presence of bone marrow edema (on STIR sequences) or osteitis (on T1 post gadolinium) in the typical anatomical areas (subchondral or periarticular bone marrow) was considered essential for defining active sacroiliitis (1). Thus MRI is currently the best imaging method for the detection of active sacroiliitis and a reduction of active inflammation has been demonstrated in randomized controlled trials with TNF alpha blockers in axial SpA patients, including patients in non-radiographic stage (7-9). In fact infliximab is effective for reducing clinical indices of disease activity and disability (assessed by ASAS response criteria, BASDAI, BASFI) as well as total MRI score and the number of sacroiliac joint MRI lesions from week 0 to week 16 (7). In patients with early axial SpA, with a symptom duration of less than 5 years, active inflammatory lesions of spine and sacroiliac joints, detected by whole-body MRI were reduced significantly more in etanercept versus sulfasalazine treated patients at weeks 48 (8). Moreover, MRI improvement of inflammatory lesions correlated with a good clinical response in patients treated with etanercept (8). A randomised placebo-controlled trial in patients with non-radiographic axial $\mathrm{SpA}$ showed that adalimumab significantly improved clinical indices and reduced inflammation in the spine and sacroiliac joints on MRI assessment after 12 weeks (9).

Our experience with non-radiographic axial SpA patient suggests that CZP could improve clinical symptoms and reduce inflammation, detected by MRI, at sacroiliac joint and entheseal level, as well as other TNF blockers (7-9). Moreover, our case also suggests that shorter disease duration, together with young age, could be predictors for a major treatment response (11). Another important aspect of this case is the dramatic and rapid improvement after the first administration of CZP, according to the evidence that clinical and functional benefits of CZP treatment in rheumatoid arthritis patients occur early, within the first week of therapy $(12,13)$. In fact, a recently post-hoc analysis on the kinetics of CZP response showed that this new molecule acts rapidly and the clinical response achieved at week 6 was a good predictor of remission at week 52 (14).

In conclusion, we suggest that CZP could be considered useful in axial $\mathrm{SpA}$ treatment in non-radiographic stage, supporting that an early treatment could play a relevant role in disease management. Nevertheless these good clinical results in our patient need the demonstration that radiographic damage could be prevented by this therapeutic approach. For this reason, randomized controlled trials with CZP in early axial $\mathrm{SpA}$, including patients in nonradiographic stage, are required in order to define clinical and pharmaeconomic impact of early anti-TNF alpha treatment on the course of the disease.

\section{REFERENCES}

1. Rudwaleit M, Jurik AG, Hermann KG, et al. Defining active sacroiliitis on magnetic resonance imaging (MRI) for classification of axial spondyloarthritis: a consensual approach by the ASAS/OMERACT MRI group. Ann Rheum Dis. 2009; 68: 1520-7.

2. Rudwaleit M, van der Heijde D, Landewé R, et al. The development of assessment of spondyloarthritis international society classification criteria for axial spondyloarthritis (part II): validation and final selection. Ann Rheum Dis. 2009; 68: 777-83.

3. Brandt J, Khariouzov A, Listing J, et al. Successful short term treatment of patients with severe undifferentiated spondyloarthritis with the anti-tumor necrosis factor- fusion receptor protein etanercept. J Rheumatol. 2004; 31 : 531-8.

4. Van den Bosch F, Kruithof E, Baeten D, et al. Effects of a loading dose regimen of three infusions of chimeric monoclonal antibody to tumor necrosis factor (infliximab) in spon- 
dyloarthropathy: an open pilot study. Ann Rheum Dis. 2000; 59: 428-33.

5. Spadaro A, Punzi L, Marchesoni A, et al. Switching from infliximab or etanercept to adalimumab in resistant or intolerant patients with spondyloarthritis: a 4-year study. Rheumatology (Oxford). 2010; 49: 1107-11.

6. Haibel H, Rudwaleit M, Listing J, et al. Efficacy of adalimumab in the treatment of axial spondylarthritis without radiographically defined sacroiliitis: results of a twelve-week randomized, double-blind, placebo-controlled trial followed by an open-label extension up to week fifty-two. Arthritis Rheum. 2008; 58: 1981-91.

7. Barkham N, Keen HI, Coates LC, et al. Clinical and imaging efficacy of infliximab in HLA-B27-Positive patients with magnetic resonance imaging-determined early sacroiliitis. Arthritis Rheum. 2009; 60: 946-54.

8. Song IH, Althoff CE, Haibel H, et al. Frequency and duration of drug-free remission after 1 year of treatment with etanercept versus sulfasalazine in early axial spondyloarthritis: 2 year data of the ESTHER trial. Ann Rheum Dis. 2012; 71: 1212-5.

9. Sieper J, van der Heijde D, Dougados M, et al. Efficacy and safety of adalimumab in patients with non-radiographic axial spondyloarthritis: results of a randomized placebo-controlled trial (ABILITY-1). Ann Rheum Dis. 2012; 72 : 815-22.

10. van der Linden S, Valkenburg HA, Cats A. Evaluation of diagnostic criteria for ankylosing spondylitis. A proposal for modification of the New York criteria. Arthritis Rheum. 1984; 27: 361-8.

11. Rudwaleit M, Listing J, Brandt J, et al. Prediction of a major clinical response (BASDAI 50) to tumour necrosis factor alpha blockers in ankylosing spondylitis. Ann Rheum Dis. 2004; 63: 665-70.

12. Mease PJ. Certolizumab pegol in the treatment of rheumatoid arthritis: a comprehensive review of its clinical efficacy and safety. Rheumatology. 2011; 50: 261-70.

13. Lubrano E, Spadaro A. Certolizumab: efficacy and safety profile of a novel pegylated TNFalpha blocking agent. Acta Biomed. 2011; 82: 26-34.

14. Keystone EC, Curtis JR, Fleischmann RM, et al. Rapid improvement of sign and symptoms of Rheumatoid Arthritis following certolizumab pegol treatment predicts longterm outcomes: post-hoc analysis of a randomized controlled trial. J Rheumatol. 2011; 38: 990-6. 